\title{
Saúde Oral - Fatores de Não Adesão aos Cheques-Dentista: Um Estudo de Caso-Controlo
}

\section{Oral Health - Factors of Non-Adherence to Dental Vouchers: A Case-Control Study}

\author{
Rita FILIPE $\triangle 1$, Pedro AGUIAR ${ }^{2}$ \\ Acta Med Port 2018 Jun;31(6):303-311 - https://doi.org/10.20344/amp.9640
}

\begin{abstract}
RESUMO
Introdução: O cheque-dentista foi criado para efetivar o acesso às consultas de medicina dentária, no entanto, a utilização dos primeiros cheques-dentistas pelos alunos com cáries de 7, 10 e 13 anos das escolas públicas da área geodemográfica do Agrupamento de Centros de Saúde Lisboa Ocidental e Oeiras (ACES LOO) tem sido baixa (23\%, ano letivo 2014/2015). Com este trabalho, pretendemos estudar os fatores associados à não utilização.

Material e Métodos: Realizámos um estudo caso-controlo que envolveu 270 alunos (135 casos e 135 controlos), provenientes das 35 escolas públicas que aceitaram participar no estudo. Foi feita uma análise descritiva dos motivos da não adesão e a pesquisa da associação entre as variáveis e a utilização/não utilização do cheque-dentista por análise estatística bivariável e multivariável, adotando-se um nível de significância de 0,05.
\end{abstract}

Resultados: Os principais motivos para a não utilização elencados $(n=135)$ foram ter dentista particular não aderente ao chequedentista $(23,7 \%)$ e ter-se esquecido de utilizar o cheque-dentista, deixando ultrapassar o prazo de validade $(21,5 \%)$. O principal fator associado à não utilização foi o aluno ser vigiado por dentista particular (OR ajustado 2,004, $p=0,017$; IC 95\%: 1,176 - 3,413) e o principal fator associado à utilização foi o encarregado de educação ter conhecimento de dentista aderente ao cheque-dentista (OR ajustado 0,096, $p<0,001$; IC 95\%: 0,047 - 0,198).

Discussão: Os resultados obtidos realçam a necessidade de se melhorar a acessibilidade aos cheques-dentista.

Conclusão: Espera-se que a identificação destes fatores possa contribuir para planear estratégias e atividades com vista a aumentar a utilização dos cheques-dentista.

Palavras-chave: Avaliação de Programas e Projetos de Saúde; Educação em Saúde Oral; Portugal; Promoção da Saúde; Saúde Oral

\section{ABSTRACT}

Introduction: A dental voucher was created to facilitate the access to oral health care, however the use of these vouchers, by students aged 7,10 and 13 with dental caries from state schools within the geodemographic area covered by the Community Health Center Group of Western Lisbon and Oeiras, had a low uptake (23\%, school year 2014/2015) The aim of this study was to examine the factors associated with this non-use.

Material and Methods: A case-control study was carried out involving 270 students ( 135 cases and 135 controls) from 35 state schools who agreed to participate in the study. A descriptive analysis of the reasons for non-adhesion to the voucher, and a study associating the variables and the use/non-use of the dental vouchers using bivariate and multivariate statistical analysis was made adopting a significance of 0.05 .

Results: The main reasons for non-use $(n=135)$ were the use of private dentists outside the dental voucher scheme $(23.7 \%)$ and forgetting to use the vouchers or exceeding the expiry date $(21.5 \%)$. The main factor associated with the non-use was students having a private dentist (OR adjusted 2.004, $p=0.012$; IC 95\%: $1.176-3.413$ ) and the main factor associated with the use was having information of dentists accepting dental vouchers (OR adjusted 0.096, $p=<0.001$; IC 95\%: $0.047-0.198$ ).

Discussion: Our findings highlight the need to improve the accessibility to dental vouchers.

Conclusion: It is hoped that the identification of these factors will contribute in the planning of strategies and activities to improve the use of dental vouchers.

Keywords: Health Education, Dental; Health Promotion; Oral Health; Portugal; Program Evaluation

\section{INTRODUÇÃO}

Segundo o estudo Global Burden of Disease de 2010, as doenças da cavidade oral afetaram 3,9 mil milhões de pessoas e provocaram a perda de 15 milhões de disability-adjusted life years (DALY) a nível mundial. ${ }^{1}$ Além disso, os custos inerentes a estas doenças são elevados, sendo considerada pela Organização Mundial da Saúde (OMS) o quarto grupo de patologias mais caras de tratar. ${ }^{2} \mathrm{Em} 2012$, só na União Europeia, os custos foram estimados em 79 mil milhões de euros. ${ }^{3}$

A cárie dentária constitui a doença crónica mais prevalente a nível mundial, ${ }^{1}$ e apesar do seu declínio global continua a ser um problema de saúde pública importante, afetando $60 \%$ - 90\% das crianças em idade escolar e a maioria dos adultos visto o seu caráter cumulativo, levando a milhões de dias de trabalho perdidos. ${ }^{2}$

A estratégia europeia e as metas definidas para a saúde oral, pela OMS, apontam para que, no ano 2020, pelo menos $80 \%$ das crianças com 6 anos estejam livres de cárie. ${ }^{4}$ Em Portugal, apesar da prevalência da cárie dentária ter vindo a diminuir, segundo os dados do III Estudo Nacional de Prevalência das Doenças Orais de 2015, apenas 55\% das crianças portuguesas com seis anos se encontravam

1. Unidade de Saúde Pública. Agrupamento de Centros de Saúde Lisboa Ocidental e Oeiras. Oeiras. Portugal.

2. Escola Nacional de Saúde Pública. Universidade Nova de Lisboa. Lisboa. Portugal.

$\triangle$ Autor correspondente: Rita Filipe. rita.filipe@arslvt.min-saude.pt

Recebido: 03 de setembro de 2017 - Aceite: 02 de maio de 2018 | Copyright @ Ordem dos Médicos 2018 
livres de cárie, valor que desce para os $32,4 \%$ no caso dos jovens com 18 anos. ${ }^{5}$

A cárie dentária tem uma elevada vulnerabilidade, com custos económicos reduzidos e ganhos em saúde relevantes, se adequadamente prevenida e precocemente tratada. ${ }^{4} \mathrm{~A}$ literatura demonstra que as intervenções dentárias preventivas que incluam cuidados rotineiros precoces, flúor e selantes, são custo efetivas. No entanto, o acesso aos cuidados de saúde oral preventiva está fortemente condicionado por falta de uma política de saúde efetiva. ${ }^{3}$

Em Portugal, a saúde oral tem sido uma das áreas onde a cobertura pública das necessidades em saúde menos se tem feito sentir. ${ }^{6}$ De acordo com os dados do European Union Statistics on Income and Living Conditions survey de 2011 (EU-SIL), Portugal situava-se entre os três países da Europa com necessidades de exame dentário não satisfeitas mais elevadas (14,5\%), sendo o principal motivo de não ir ao dentista o elevado preço. ${ }^{7}$

De forma a colmatar esta carência, foi implementado em 2005, o Programa Nacional de Promoção de Saúde Oral (PNPSO) ${ }^{6}$ e em 2008, de forma a efetivar o acesso às consultas de medicina dentária, foi criado o modelo do cheque-dentista. ${ }^{8}$

As crianças e jovens (7, 10 e 13 anos) são o principal grupo beneficiário, representando $85 \%$ do total dos cheques-dentista emitidos. ${ }^{6}$ Às crianças e jovens de 7 e 10 podem ser atribuídos até dois cheques-dentista por ano letivo e às de 13 anos podem ser atribuídos até três cheques-dentista por ano letivo, tendo cada cheque o valor máximo de 35 euros. $^{9}$

Cabe à equipa de saúde oral da Unidade de Saúde Pública ir às escolas públicas e fazer uma triagem aos alunos: sendo feita a emissão do cheque-dentista no caso das crianças terem cáries em dentes permanentes, no caso de não terem cáries é emitido um documento de acesso a consulta de higiene oral. ${ }^{10}$ Neste grupo etário, apesar do número de primeiros cheques-dentista utilizados ter vindo a aumentar desde 2009 a 2013, de 188263 para 312 394, ${ }^{11}$ a proporção de utilização situou-se, a nível nacional, apenas nos $64 \%{ }^{6}$

O Agrupamento de Centros de Saúde Lisboa Ocidental e Oeiras (ACES LOO) abrange um total de 233465 habitantes, ${ }^{12}$ numa área maioritariamente urbana que possui uma densidade populacional e índice de dependência de jovens e de idosos superior à verificada na Administração Regional de Saúde de Lisboa e Vale do Tejo (ARS LVT), assim como um maior nível de escolaridade e de poder de compra e uma menor taxa de desemprego. ${ }^{13}$ O ACES LOO pertence à ARS LVT, que é a ARS que apresenta, a nível nacional, um menor rácio de médicos dentistas aderentes ao cheque-dentista. ${ }^{6} \mathrm{Em}$ termos de utilização do cheque-dentista no ACES LOO, verifica-se uma baixa proporção de utilização (23\% no ano letivo $2014 / 2015){ }^{14}$

A literatura mostra que fatores como o nível de escolaridade, socioeconómico e de literacia dos pais; o nível de acesso a informação e recursos; e o acesso a cuidados de saúde oral têm influência na saúde oral das crianças e jovens. ${ }^{15}$

Sendo o cheque-dentista gratuito e dirigido a crianças com necessidades dentárias já identificadas, com este estudo pretendemos conhecer os fatores associados à não adesão ao primeiro cheque-dentista, nos alunos de 7, 10 e 13 anos, inscritos nas escolas públicas da área geodemográfica do ACES LOO, durante o ano letivo 2014/2015.

Os objetivos específicos deste estudo consistem na comparação de casos e controlos face às seguintes variáveis: caracterização sociodemográfica do encarregado de educação (idade, sexo, grau de parentesco, estado civil, nível de escolaridade, profissão, situação profissional e, se aplicável, motivo de inatividade), caracterização sociodemográfica do aluno (idade, sexo, escola de inscrição) e análise de variáveis relativas ao PNPSO (recetor do cheque-dentista, conhecimento de dentista aderente ao cheque-dentista, vigilância por dentista particular e se sim, se este era aderente ao cheque-dentista).

\section{MATERIAL E MÉTODOS}

Realizámos um estudo epidemiológico observacional caso-controlo retrospetivo. A população-alvo correspondeu aos alunos dos grupos etários dos 7, 10 e 13 anos inscritos nas escolas públicas da área geodemográfica do ACES LOO que receberam o primeiro cheque-dentista no ano letivo 2014/2015 (total de alunos elegíveis: 2496). Definimos como caso o aluno de 7, 10 ou 13 anos, inscrito em escola pública da área geodemográfica do ACES LOO, que tenha recebido o primeiro cheque-dentista e que não o tenha utilizado, no ano letivo 2014/2015 e como controlo o aluno de 7, 10 ou 13 anos, inscrito em escola pública da área geodemográfica do ACES LOO, que tenha recebido o primeiro cheque-dentista e que o tenha utilizado, no ano letivo 2014/2015.

Para participação no estudo, considerámos os seguintes critérios de inclusão: tratar-se de uma criança com 7, 10 ou 13 anos no ano letivo 2014/2015, estar inscrita nas escolas públicas da área geodemográfica do ACES LOO no ano letivo 2014/2015, ter recebido o primeiro cheque-dentista no ano letivo 2014/2015 e ter o consentimento informado do encarregado de educação (EE) para participação no estudo (Apêndice 1: https://www.actamedicaportuguesa.com/revista/index.php/ amp/article/view/9640/5447).

Como critérios de exclusão considerámos: ter participado no estudo-piloto, o EE não ter recebido o cheque-dentista no ano letivo 2014/2015, o EE não ter assinado o consentimento informado e o EE não ter respondido ao questionário.

Para a realização deste estudo foram tidas em consideração as questões éticas e morais previstas na legislação portuguesa (Lei no $67 / 98$ de 26 de agosto), ${ }^{16}$ tendo sido dado o parecer positivo quer da Comissão de Ética da ARS LVT (número de autorização 9136/CES/ 2016) (Apêndice 2: https://www.actamedicaportuguesa.com/revista/index. php/amp/article/view/9640/5448), quer da Diretora Executiva do ACES LOO (Apêndice 3: https://www. 
actamedicaportuguesa.com/revista/index.php/amp/article/ view/9640/5449).

Após termos a aprovação da Comissão de Ética da ARS LVT, foi enviado um email pela Gestora do Programa de Saúde Escolar do ACES LOO a todos os diretores das escolas públicas da área geodemográfica do ACES a explicar o estudo e a solicitar participação. Do total de 62 escolas [14 Agrupamentos de Escolas (AE) e 4 Escolas Não Agrupadas], aceitaram participar no estudo 35 escolas (11 AE e 1 Escola Não Agrupada) (Apêndice 4: https://www. actamedicaportuguesa.com/revista/index.php/amp/article/ view/9640/5450, o que perfez uma percentagem de adesão das escolas de $56,5 \%$.

Numa segunda fase, das 35 escolas que aceitaram participar no estudo, realizámos o cruzamento das listas dos alunos que utilizaram o primeiro cheque-dentista no ano letivo 2014/2015, através do Sistema de Informação em Saúde Oral com a lista dos alunos matriculados no presente ano letivo, de forma a excluir alunos que tenham, entretanto, mudado de escola e obteve-se um total de 884 alunos elegíveis: 450 casos e 434 controlos. Foi enviado um questionário e um consentimento informado ao total de 884 alunos elegíveis.

Destas 35 escolas, foi feita uma reunião com o professor responsável pelos projetos de educação para a saúde (PES) de cada escola a explicar o estudo, os quais por sua vez articularam os diretores de turma dos alunos elegíveis para participar no estudo. Cada professor responsável peIos PES funcionou como ele de ligação da escola com os investigadores.

Para o cálculo da amostra necessária utilizámos a fórmula de cálculo de amostras para estudos caso-controlo, considerando um nível de significância de $5 \%$, poder de teste de $80 \%$, rácio caso-controlo $1: 1$, odds ratio $(\mathrm{OR})$ de 2,5 e uma proporção de exposição fatorial de 50\% nos controlos. A dimensão amostral teórica necessária seria de 162 alunos (81 casos e 81 controlos). ${ }^{17}$ No entanto, tendo em conta a literatura, considerámos uma taxa de resposta de $20 \%,{ }^{18}$ pelo que seria necessário abranger pelo menos 810 alunos ( 405 casos e 405 controlos) para obter o valor teórico calculado para a amostra (162). Além disso, ainda seria necessário abranger mais alunos, visto ter que se realizar um pré-teste. Deste modo, decidimos selecionar todos os alunos elegíveis para o estudo (884), tendo sido enviado um questionário e um consentimento informado a todos os alunos elegíveis, sendo estes preenchidos pelo EE.

O questionário (Apêndice 5: https://www.actamedicaportuguesa.com/revista/index.php/amp/article/ view/9640/5451) foi baseado na literatura e era constituído por três partes: a primeira parte era referente ao PNPSO (recetor do cheque-dentista, conhecimento de dentista aderente, vigilância por dentista particular e se sim, se este era aderente ao cheque-dentista), a segunda parte era referente à caracterização sociodemográfica aluno (idade, sexo, escola de inscrição) e a terceira parte à caracterização sociodemográfica do EE (idade, sexo, grau de parentesco, estado civil, nível de escolaridade, profissão, ${ }^{19}$ situação profissional e, se aplicável, motivo de inatividade) (Apêndice 6: https://www.actamedicaportuguesa.com/revista/index.php/amp/article/view/9640/5452). Considerámos como variável dependente a não utilização do primeiro cheque dentista. Para os casos, foram analisados os motivos da não utilização do cheque-dentista. Para aferir a validade facial do questionário foi realizado um pré-teste a $10 \mathrm{EE}$ selecionados aleatoriamente, do qual não resultaram alterações.

Do total de questionários enviados, obtivemos 362 respostas (percentagem global de resposta de $41 \%$; min. máx.: $20 \%$ - 56,1\%). Após aplicação dos critérios de inclusão e exclusão, foram considerados 270 respostas válidas das quais 135 correspondiam a casos e 135 a controlos (Fig. 1). Os dados obtidos foram analisados no programa IBM SPSS Statistics ${ }^{\circledR}$ versão 20.0.

62 Escolas foram convidadas a participar no Estudo (total de alunos elegíveis: 2496)

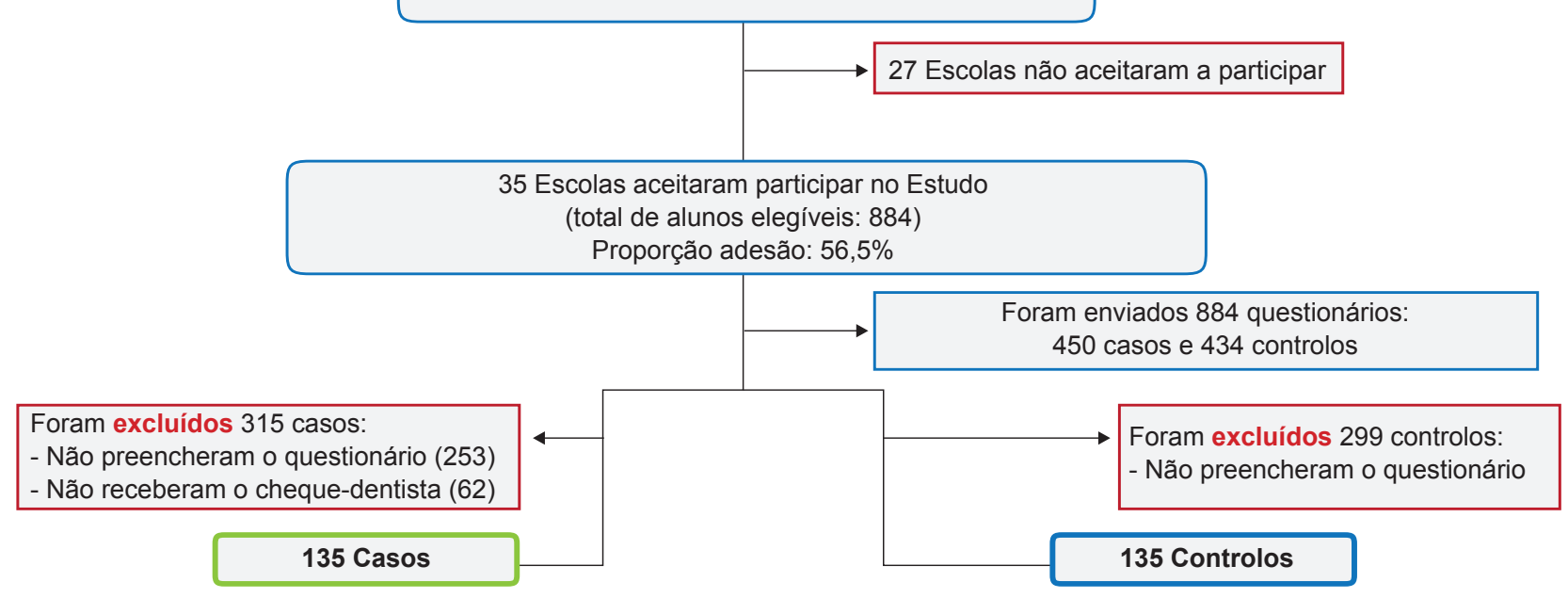

Figura 1 - Fluxograma do estudo 
Na análise bivariável, foram comparados dois grupos, os casos e os controlos, face a uma série de variáveis, entre as quais se encontravam variáveis numéricas e categóricas. $\mathrm{Na}$ avaliação dos dois grupos face a variáveis categóricas, fizemos a análise através do teste do qui-quadrado ou, quando não aplicável, através do teste exato de Fisher, enquanto que para as variáveis numéricas utilizámos o teste $t$ de Student para amostras independentes ou o teste de Mann-Whitney caso não se tenha verificado o pressuposto de normalidade. Calculámos o valor $p$, para cada variável independente em estudo e analisámos a homogeneidade dos casos face aos controlos. ${ }^{20}$

Foram elaboradas tabelas de cruzamento contendo frequências absolutas, relativas e respetivos intervalos de confiança (IC) a 95\%, para cada um dos grupos. As medidas de associação pesquisadas foram o cálculo do OR e respetivo IC para um nível de confiança de $95 \%$ e foi avaliada a magnitude e o sentido da associação. Para as variáveis numéricas, a magnitude da associação foi calculada através da diferença de médias e respetivo intervalo de confiança, enquanto para as variáveis categóricas foi calculada através dos OR e respetivos intervalos de confiança. Do resultado da análise bivariável, foram incluídas na análise de regressão múltipla as variáveis que cumpriram os seguintes critérios: resultados estatisticamente significativos nas relações com a variável dependente (valor $p$ $<0,05$ ); variáveis que apesar de não revelarem resultados estatisticamente significativos nas relações com a variável dependente originem valores $p<0,20$; variáveis que apesar de não revelarem resultados estatisticamente significativo, tenham uma especial importância para a investigação. ${ }^{20}$

$\mathrm{Na}$ análise multivariável, aplicou-se o modelo de regressão logística multivariável o que nos permitiu eliminar possíveis fatores de confundimento. A magnitude das associações foi obtida pelo cálculo do valor exponencial dos coeficientes de regressão, resultando nos OR ajustados entre si. Para se chegar ao valor final de cada OR ajustado para cada variável, ao longo do processo de análise multivariada, foi retirada, de cada vez, a variável com maior valor $p$, obtendo-se um modelo otimizado com uma tabela final com as variáveis cuja associação com a não adesão ao cheque-dentista foi estatisticamente significativa $(p<0,05)$. Foram excluídas da análise, as variáveis cujos missing values foram superiores a $10 \% .^{20}$

Para análise da qualidade de ajustamento do modelo de regressão logística, utilizámos a área sob a receiver operator characteristic curve (ROC). A curva ROC permitiu-nos ver até que ponto as probabilidades estimadas pelo modelo de regressão logística discriminaram os casos de não utilização do cheque-dentista. ${ }^{20}$

Os resultados do modelo otimizado da regressão logística foram confirmados através de modelos lineares generalizados [generalized estimated equations (GEE)] para uma estrutura de correlação uniforme das observações dos alunos dentro das escolas e do modelo logístico. Após a realização da análise estatística, os questionários foram destruídos de forma a garantir a privacidade e confidencialidade dos mesmos.

\section{RESULTADOS}

Foram incluídos no estudo 135 casos e 135 controlos. A percentagem global de questionários excluídos pelo facto de os EE não terem recebido o cheque-dentista foi de $17 \%$ $(n=62)$, variando nas escolas/AE participantes entre $0 \%$ $33 \%$, o que o que introduz um viés importante na análise da baixa utilização do mesmo.

Os principais motivos elencados pelos encarregados de educação para a não utilização do cheque-dentista ( $\mathrm{n}$ = 135), no ano letivo 2014/2015, foram: ter dentista particular que não era aderente ao cheque-dentista $(23,7 \%)$ e ter-se esquecido de utilizar o cheque-dentista, deixando ultrapassar o prazo de validade (21,5\%). Salientamos que $10,4 \%$ referiram não ter conseguido localizar médicos aderentes ao cheque-dentista (Tabela 1).

Considerando a caracterização sociodemográfica dos alunos (Tabela 2), verificámos que tanto nos casos e controlos, o sexo feminino foi ligeiramente mais frequente (53,3\% vs $53,3 \%)$ e a maior proporção de cheques-dentista

Tabela 1 - Motivos da não adesão ao cheque-dentista no ano letivo 2014/2015 ( $n=135$ )

\begin{tabular}{lcc}
\hline Motivos da não adesão ao cheque-dentista & Frequência absoluta & Frequência relativa \\
\hline Não achou necessário & 20 & $14,8 \%$ \\
Esqueceu-se de usar, deixando ultrapassar o prazo de validade & 29 & $21,5 \%$ \\
Perdeu o cheque-dentista & 6 & $4,4 \%$ \\
Não conseguiu localizar médicos aderentes ao cheque-dentista & 14 & $10,4 \%$ \\
Conseguiu encontrar clínicas aderentes ao cheque-dentista, não tendo & 3 & $2,2 \%$ \\
ficado satisfeito com o atendimento & 4 & $3,0 \%$ \\
Não aderiu por aconselhamento de outros profissionais & 1 & $0,8 \%$ \\
Já usou em anos anteriores, não tendo ficado satisfeito com o atendimento & 13 & $9,6 \%$ \\
Não recebeu informação sobre como utilizar o cheque-dentista e sua & 32 & $23,7 \%$ \\
importância & 8 & $5,9 \%$ \\
Tem dentista particular que não é aderente & 5 & $3,7 \%$ \\
Tem seguro de saúde & 3 & \\
Não especificou & & \\
\hline
\end{tabular}


Tabela 2 - Apresentação comparativa dos dados referentes à análise estatística bivariável para a informação relativa ao aluno $(\mathrm{n}=270)$

\begin{tabular}{|c|c|c|c|c|c|c|}
\hline $\begin{array}{l}\text { Variável em } \\
\text { análise }\end{array}$ & Categorias & $\begin{array}{c}\text { Medidas } \\
\text { estatísticas }\end{array}$ & $\begin{array}{c}\text { Grupo dos } \\
\text { casos }^{1} \\
(n=135)\end{array}$ & $\begin{array}{c}\text { Grupo dos } \\
\text { controlos }^{2} \\
(n=135)\end{array}$ & Odds ratio; (IC 95\%) & Valor $p$ \\
\hline \multirow{2}{*}{ Sexo } & Masculino (referência) & $\mathrm{n}(\%)$ & $63(46,7 \%)$ & $63(46,7 \%)$ & \multirow{2}{*}{1,$000 ;(0,620-1,613)$} & \multirow{2}{*}{1,000} \\
\hline & Feminino & $\mathrm{n}(\%)$ & $72(53,3 \%)$ & $72(53,3 \%)$ & & \\
\hline \multirow{3}{*}{ Idade } & 7 anos (referência) & $\mathrm{n}(\%)$ & $21(15,6 \%)$ & $25(18,5 \%)$ & Referência & \multirow{3}{*}{0,572} \\
\hline & 10 anos & $\mathrm{n}(\%)$ & $60(44,4 \%)$ & $64(47,4 \%)$ & 1,$116 ;(0,566-2,200)$ & \\
\hline & 13 anos & $\mathrm{n}(\%)$ & $54(40,0 \%)$ & $46(34,1 \%)$ & 1,$398 ;(0,693-2,817)$ & \\
\hline \multirow{12}{*}{$\begin{array}{l}\text { Escola/ AE } \\
\text { que o aluno } \\
\text { frequentava } \\
\text { no ano letivo } \\
2014 / 2015\end{array}$} & AE Santa Catarina (referência) & $\mathrm{n}(\%)$ & $21(15,6 \%)$ & $25(18,5 \%)$ & Referência & \multirow{12}{*}{0,213} \\
\hline & AE Miraflores & $\mathrm{n}(\%)$ & $6(4,4 \%)$ & $14(10,4 \%)$ & 0,$510 ;(0,167-1,561)$ & \\
\hline & AE Carnaxide Portela & $\mathrm{n}(\%)$ & $11(8,1 \%)$ & $4(3,0 \%)$ & 3,$274 ;(0,907-11,810)$ & \\
\hline & AE Linda-a-Velha e Queijas & $\mathrm{n}(\%)$ & $14(10,4 \%)$ & $7(5,2 \%)$ & 2,$381 ;(0,811-6,990)$ & \\
\hline & AE São Bruno & $\mathrm{n}(\%)$ & $8(5,9 \%)$ & $11(8,1 \%)$ & 0,$866 ;(0,294-2,549)$ & \\
\hline & AE Conde de Oeiras & $\mathrm{n}(\%)$ & $3(2,2 \%)$ & $4(3,0 \%)$ & 1,$176 ;(0,179-4,446)$ & \\
\hline & AE Aquilino Ribeiro & $\mathrm{n}(\%)$ & $16(11,9 \%)$ & $17(12,6 \%)$ & 1,$120 ;(0,457-2,745)$ & \\
\hline & AE Paço de Arcos & $\mathrm{n}(\%)$ & $20(14,8 \%)$ & $24(17,8 \%)$ & 0,$992 ;(0,433-2,275)$ & \\
\hline & AE Manuel da Maia & $\mathrm{n}(\%)$ & $7(5,2 \%)$ & $8(5,9 \%)$ & 1,$042 ;(0,324-3,351)$ & \\
\hline & ES Rainha Dona Amélia & $\mathrm{n}(\%)$ & $1(0,7 \%)$ & $1(0,7 \%)$ & 1,$190 ;(0,070-20,211)$ & \\
\hline & AE Bartolmeu de Gusmão & $\mathrm{n}(\%)$ & $5(3,7 \%)$ & $8(5,9 \%)$ & 0,$744 ;(0,211-2,620)$ & \\
\hline & AE Carnaxide & n (\%) & $23(17,0 \%)$ & $12(8,9 \%)$ & 2,$282 ;(0,921-5,655)$ & \\
\hline
\end{tabular}

AE: agrupamento de escolas; ES: escola secundária; IC: intervalo de confiança

1: Aluno que não utilizou o cheque-dentista; 2: aluno que utilizou o cheque-dentista

foi entregue aos 10 anos de idade $(44,4 \%$ vs $47,4 \%)$ e a menor aos 7 anos (15,6\% vs 18,5\%).

Relativamente à Escola/AE que o aluno estava inscrito, verificámos que a menor proporção de alunos estava inscrita na Escola Secundária Rainha Dona Amélia $(0,7 \%$ vs $0,7 \%$ ) e a maior proporção de casos estava inscrita no AE de Carnaxide (17\%) e de controlos no AE Santa Catarina $(18,7 \%)$.

Não verificámos diferenças entre o grupo de casos e controlos, no que diz respeito à proporção do sexo feminino $(p=1,000)$, à proporção de idades $(7,10$ e 13 anos) em que foi entregue o primeiro cheque-dentista $(p=0,572)$ e à escola/AE que os alunos frequentavam no ano letivo $2014 / 2015(p=0,213)$. Estes dados podem nos fazer assumir a homogeneidade estatística dos grupos em comparação, para o nível de significância de 0,05.

Considerando a caracterização sociodemográfica dos EE (Tabela 3), verificámos que, tanto nos casos como nos controlos, o sexo feminino foi o predominante $(83,7 \%$ vs $83,0 \%, p=0,870)$ e a idade média foi semelhante $(41,68 \pm$ $8,39$ vs 40,39 $\pm 6,75, p=0,166)$.

Na maioria das vezes, o encarregado de educação correspondeu à mãe do aluno $(81,5 \%$ vs $81,5 \%, p=0,803)$, encontrava-se casado/união de facto $(66,7 \%$ vs $58,5 \%, p$ $=0,225)$, empregado $(77,8 \%$ vs $78,5 \%, p=0,833)$, desenvolvendo trabalho qualificado $(82,7 \%$ vs $81,1 \%, p=0,769)$. Quando questionados pelos motivos de estarem inativos, o desemprego foi o principal motivo, sendo este menos elevado nos EE dos casos (63,3\% vs $82,8 \%, p=0,318$ ).

Em termos de nível de escolaridade, $35,1 \%$ dos EE dos casos e $23,7 \%$ dos EE dos controlos tinham completado

o ensino superior, sendo esta diferença estatisticamente significativa $(p=0,041)$. Verificámos uma associação positiva entre ter completado o ensino superior e a não utilização do cheque-dentista (OR = 1,739; IC 95\%: 1,021 - 2,961).

Considerando a informação relativa à vigilância de Saúde Oral e aos cheques-dentista (Tabela 4), verificou-se que o cheque-dentista foi entregue maioritariamente ao aluno (75,8\% vs 85,8\%, $p=0,092)$.

O conhecimento do EE de dentista aderente ao cheque-dentista foi muito inferior nos casos $(14,1 \%$ vs $62,2 \%$ $p<0,001$ ) e associou-se a uma redução relativa da odd (possibilidade de ocorrência) da não utilização do cheque-dentista em 90,1\% [OR = 0,099, IC 95\%: 0,055 - 0,181]. Por sua vez, a vigilância do aluno por dentista particular no ano letivo $2014 / 2015$ foi superior nos casos $(67,4 \%$ vs $53,3 \%, p=0,018$ ) e associou-se a um aumento relativo da odd da não utilização do cheque-dentista em $81.0 \%$ (OR = 1,810, IC 95\%: 1,104 - 2,965). Salientamos que cerca de $1 / 3$ dos alunos que não utilizaram o cheque-dentista também não foram seguidos por dentista particular.

Quando questionámos sobre se o dentista particular era aderente ao cheque-dentista verificámos que apenas o era em $8,8 \%(n=8)$ dos casos e em $43,8 \%$ dos controlos ( $n$ $=32$ ). Esta diferença foi estatisticamente significativa ( $p<$ $0,001)$, estando a vigilância por dentista aderente ao cheque-dentista associada a uma redução relativa da odd da não utilização do cheque-dentista em $87,3 \%$ (OR = 0,123, IC 95\%: 0,054-0,302).

O modelo de regressão obtido foi estatisticamente significativo (teste Omnibus $<0,001$ ), com uma boa qualidade de ajustamento (área sob a curva ROC 77,8\%, $p<0,001$, 
Tabela 3 - Apresentação comparativa dos dados referentes à análise estatística bivariável para a informação relativa aos EE $(n=270)$

\begin{tabular}{|c|c|c|c|c|c|c|}
\hline $\begin{array}{l}\text { Variável em } \\
\text { análise }\end{array}$ & Categorias & $\begin{array}{l}\text { Medidas } \\
\text { estatísticas }\end{array}$ & $\begin{array}{l}\text { Grupo dos } \\
\text { casos }^{1} \\
(n=135)\end{array}$ & $\begin{array}{l}\text { Grupo dos } \\
\text { controlos }^{2} \\
(n=135)\end{array}$ & Odds ratio; IC (95\%) & Valor $p$ \\
\hline \multirow{2}{*}{ Sexo } & Masculino (referência) & n (\%) & $22(16,3 \%)$ & $23(17,0 \%)$ & \multirow{2}{*}{1,$055 ;(0,556-2,001)$} & \multirow{2}{*}{0,870} \\
\hline & Feminino & $\mathrm{n}(\%)$ & $113(83,7 \%)$ & $112(83,0 \%)$ & & \\
\hline Idade & $\begin{array}{l}\text { Média } \\
\text { Mediana } \\
\text { Desvio - Padrão } \\
\text { Mínimo - Máximo }\end{array}$ & NA & $\begin{array}{c}41,68 \\
41,00 \\
8,39 \\
23-78\end{array}$ & $\begin{array}{c}40,39 \\
40,00 \\
6,75 \\
23-66\end{array}$ & $\begin{array}{c}-1,289 ; \\
\text { (Diferença de médias) }\end{array}$ & 0,166 \\
\hline \multirow{2}{*}{$\begin{array}{l}\text { Idade } \\
\text { recodificado }\end{array}$} & < 45 anos (referência) & n (\%) & $96(71,1 \%)$ & $93(68,9 \%)$ & \multirow{2}{*}{0,$900 ;(0,534-1,514)$} & \multirow{2}{*}{0,690} \\
\hline & $\geq 45$ anos & $\mathrm{n}(\%)$ & $39(28,9 \%)$ & $42(31,1 \%)$ & & \\
\hline \multirow{3}{*}{$\begin{array}{l}\text { Grau de } \\
\text { parentesco } \\
\text { do EE }\end{array}$} & Mãe (referência) & n (\%) & $110(81,5 \%)$ & $110(81,5 \%)$ & Referência & \multirow{3}{*}{0,803} \\
\hline & Pai & $\mathrm{n}(\%)$ & $18(13,3 \%)$ & $20(14,8 \%)$ & 0,$900 ;(0,452-1,793)$ & \\
\hline & Outro & $\mathrm{n}(\%)$ & $7(5,2 \%)$ & $5(3,7 \%)$ & 1,$400 ;(0,431-4,546)$ & \\
\hline \multirow{4}{*}{ Estado civil } & $\begin{array}{l}\text { Casado/união de facto } \\
\text { (referência) }\end{array}$ & $\mathrm{n}(\%)$ & $90(66,7 \%)$ & $79(58,5 \%)$ & Referência & \multirow{4}{*}{$0,225^{*}$} \\
\hline & Solteiro & n (\%) & $28(20,7 \%)$ & $31(23,0 \%)$ & 0,$793 ;(0,438-1,435)$ & \\
\hline & Divorciado/separado & n (\%) & $14(10,4 \%)$ & $24(17,8 \%)$ & 0,$512 ;(0,248-1,057)$ & \\
\hline & Viúvo & n (\%) & $3(2,2 \%)$ & $1(0,7 \%)$ & 2,$633 ;(0,268-25,829)$ & \\
\hline \multirow[b]{2}{*}{ Empregado } & Não (referência) & $\mathrm{n}(\%)$ & $30(22,2 \%)$ & $29(21,5 \%)$ & \multirow[b]{2}{*}{0,$958 ;(0,538-1,706)$} & \multirow[b]{2}{*}{0,833} \\
\hline & Sim & $\mathrm{n}(\%)$ & $105(77,8 \%)$ & $106(78,5 \%)$ & & \\
\hline \multirow{5}{*}{$\begin{array}{l}\text { Motivos de } \\
\text { não estar } \\
\text { empregado }\end{array}$} & Desempregado (referência) & $\mathrm{n}(\%)$ & $19(63,3 \%)$ & $24(82,8 \%)$ & Referência & \multirow{5}{*}{$0,318^{*}$} \\
\hline & Reformado & $\mathrm{n}(\%)$ & $3(11,4 \%)$ & $1(3,4 \%)$ & 3,$789 ;(0,364-39,412)$ & \\
\hline & Estudante & $\mathrm{n}(\%)$ & $1(2,9 \%)$ & $0(0,0 \%)$ & 0,$442 ;(0,316-0,618)$ & \\
\hline & Doméstico & $\mathrm{n}(\%)$ & $6(20,0 \%)$ & $2(6,9 \%)$ & 3,$789 ;(0,686-20,946)$ & \\
\hline & Outro & $\mathrm{n}(\%)$ & $1(2,9 \%)$ & $2(6,9 \%)$ & 0,$632 ;(0,053-7,502)$ & \\
\hline \multirow{2}{*}{$\begin{array}{l}\text { Nivel de } \\
\text { escolaridade }^{1}\end{array}$} & $\begin{array}{l}\text { Ensino Primário a Secundário } \\
\text { (referência) }\end{array}$ & $\mathrm{n}(\%)$ & $87(64,9 \%)$ & $103(76,3 \%)$ & \multirow[t]{2}{*}{1,$739 ;(1,021-2,961)$} & \multirow[t]{2}{*}{0,041} \\
\hline & Ensino Superior & $\mathrm{n}(\%)$ & $47(35,1 \%)$ & $32(23,7 \%)$ & & \\
\hline \multirow[t]{2}{*}{ Profissão ${ }^{2}$} & $\begin{array}{l}\text { Trabalhadores não qualificados } \\
\text { (referência) }\end{array}$ & $\mathrm{n}(\%)$ & $18(17,3 \%)$ & $20(18,9 \%)$ & \multirow[t]{2}{*}{1,$111 ;(0,550-2,245)$} & \multirow[t]{2}{*}{0,769} \\
\hline & Trabalhadores qualificados & n (\%) & $86(82,7 \%)$ & $86(81,1 \%)$ & & \\
\hline
\end{tabular}

AE: agrupamento de escolas; EE: encarregado de educação; ES: escola secundária; IC: intervalo de confiança; NA: não aplicável; 1 : Casos $n$ = 134; 2: Casos n = 104

* Utilização do Teste Exacto de Fisher; '1: Aluno que não utilizou o cheque-dentista; ${ }^{2}$ : Aluno que utilizou o cheque-dentista

IC 95\%: 0,721 - 0,834), acertando em $74,1 \%$ das previsões. Após otimização do modelo, este passou a incluir as variáveis referentes ao conhecimento de dentista aderente ao cheque-dentista e à vigilância por dentista particular no ano letivo 2014/2015. Posteriormente, realizou-se uma modelação multivariada final confirmatória entrando em conta com uma estrutura de correlação uniforme dentro das escolas no modelo de regressão logística, utilizando as variáveis da modelação de regressão logística multivariável otimizada. Os resultados da modelação multivariada foram semeIhentes no que diz respeito às estimativas de efeito $(\mathrm{OR})$ já apresentadas, assim como, a sua significância estatística (Tabela 5).

Da análise destas variáveis verificámos que o conhecimento de dentista aderente ao cheque-dentista está associado a uma maior utilização do cheque-dentista (redução relativa da odd de não utilizar o cheque-dentista em $90,4 \%$, OR ajustado 0,096, $p<0,001$ ). Por outro lado, a vigilância do aluno por dentista particular está associado a uma menor utilização do cheque-dentista, havendo um aumento relativo de $100 \%$ na odd da não utilização do cheque-dentista (OR ajustado 2,004, $p=0,012$ ).

\section{DISCUSSÃO}

Os resultados do nosso estudo demonstraram que o principal fator associado à baixa adesão ao cheque-dentista deve-se ao baixo conhecimento que os EE têm de dentistas aderentes ao cheque-dentista e ao facto dos alunos já serem seguidos por dentista particular. Contudo um número significativo de alunos, cerca de $1 / 3$, não utilizou o cheque-dentista nem foi seguido por dentista particular, perdendo-se uma oportunidade para a prestação de cuida- 
Tabela 4 - Apresentação comparativa dos dados referentes à análise estatística bivariável para a informação relativa ao PNPSO ( $\mathrm{n}=270$ )

\begin{tabular}{|c|c|c|c|c|c|c|}
\hline Variável em análise & Categorias & $\begin{array}{l}\text { Medidas } \\
\text { estatísticas }\end{array}$ & $\begin{array}{c}\text { Grupo dos } \\
\text { casos }^{1} \\
(n=135)\end{array}$ & $\begin{array}{c}\text { Grupo dos } \\
\text { controlos }^{2} \\
(n=135)\end{array}$ & Odds ratio; IC (95\%) & Valor $p$ \\
\hline \multirow{3}{*}{ Entrega do $1^{\circ} \mathrm{CD}$} & Aluno (referência) & $\mathrm{n}(\%)$ & $100(75,7 \%)$ & $115(85,8 \%)$ & Referência & \multirow{3}{*}{$0,092^{*}$} \\
\hline & EE & $\mathrm{n}(\%)$ & $27(20,5 \%)$ & $17(12,7 \%)$ & 1,$826 ;(0,941-3,546)$ & \\
\hline & Outra pessoa & $\mathrm{n}(\%)$ & $5(3,8 \%)$ & $2(1,5 \%)$ & 2,$275 ;(0,546-15,144)$ & \\
\hline \multirow{2}{*}{$\begin{array}{l}\text { Conhecimento de } \\
\text { Dentista aderente } \\
\text { ao CD }\end{array}$} & Não (referência) & $\mathrm{n}(\%)$ & $116(85,9 \%)$ & $51(37,8 \%)$ & \multirow{2}{*}{0,$099 ;(0,055-0,181)$} & \multirow{2}{*}{$<0,001$} \\
\hline & $\operatorname{Sim}$ & $\mathrm{n}(\%)$ & $19(14,1 \%)$ & $84(62,2 \%)$ & & \\
\hline \multirow{2}{*}{$\begin{array}{l}\text { Vigilância por } \\
\text { Dentista particular }\end{array}$} & Não (referência) & $\mathrm{n}(\%)$ & $44(32,6 \%)$ & $63(46,7 \%)$ & \multirow{2}{*}{1,$810 ;(1,104-2,965)$} & \multirow{2}{*}{0,018} \\
\hline & Sim & $\mathrm{n}(\%)$ & $91(67,4 \%)$ & $72(53,3 \%)$ & & \\
\hline \multirow{2}{*}{$\begin{array}{l}\text { Vigilância por } \\
\text { Dentista particular } \\
\text { aderente ao } C D^{3}\end{array}$} & Não (referência) & $\mathrm{n}(\%)$ & $83(91,2 \%)$ & $41(56,9 \%)$ & \multirow{2}{*}{0,$127 ;(0,054-0,302)$} & \multirow{2}{*}{$<0,001$} \\
\hline & Sim & $\mathrm{n}(\%)$ & $8(8,8 \%)$ & $31(43,1 \%)$ & & \\
\hline
\end{tabular}

CD: cheque-dentista; EE: encarregado de educação; 3: Casos $n=91$ e Controlos $n=72$

* Utilização do Teste Exacto de Fisher; 1: Aluno que não utilizou o cheque-dentista; 2: Aluno que utilizou o cheque-dentista

dos de saúde oral personalizados, preventivos e curativos, de forma gratuita.

Por outro lado, verificou-se que $17 \%$ dos alunos não utilizou o cheque-dentista por efetivamente não o ter recebido, o que demonstra um problema de acesso.

Segundo o II Barómetro de Saúde Oral realizado pela Ordem dos Médicos Dentistas cerca de $60 \%$ dos portugueses não foi ao dentista por questões monetárias. ${ }^{21} \mathrm{O}$ cheque-dentista ao ser gratuito tem como objetivo ultrapassar a barreira financeira. ${ }^{8}$ No entanto, a literatura mostra que para melhorar o acesso aos cuidados de saúde oral é necessário não reduzir apenas barreiras financeiras, mas também reduzir barreiras não financeiras, como barreiras no acesso, baixa literacia e oferta inadequada de dentistas. $^{22,23}$

A literacia em saúde oral consiste na "capacidade para obter, processar e compreender informação básica de saúde e dos serviços, de forma a tomar decisões de saúde apropriadas" ${ }^{24}$ De acordo com o estudo Literacia em Saúde, dos Dados à Ação: Tradução, Validação e Aplicação do European Health Literacy Survey em Portugal, 61\% dos portugueses apresenta um nível de literacia geral em saúde problemático ou inadequado, situando-se a média dos nove países em $49,2 \%{ }^{25} \mathrm{~A}$ baixa literacia em saúde oral dos pais está associada a uma pior saúde oral dos filhos e uma menor utilização dos serviços de saúde. ${ }^{24}$

Os nossos resultados vão ao encontro destes dados, visto $41 \%$ dos EE que não utilizaram o cheque-dentista terem referido que os motivos se deveram a não considerarem necessário $(14,8 \%)$, não terem recebido informações sobre como utilizar o cheque-dentista $(9,6 \%)$, terem-se esquecido de utilizar $(21,5 \%)$ e terem perdido o cheque-dentista $(4,4 \%)$, o que demostra uma baixa valorização da importância da saúde oral e de como utilizar os serviços de saúde.

Num estudo espanhol verificou-se que o aumento de dentistas aderentes (28 para 161) a um programa de saúde oral dirigido à população infantil se traduziu num aumento de utilização do mesmo de $37,4 \%$ para $67,2 \%$, havendo uma correlação positiva entre o aumento da extensão da rede do programa e a proporção de utilização do mesmo, assim como uma melhoria da saúde oral. ${ }^{26}$

No nosso estudo, $10,4 \%$ dos EE não utilizaram o cheque-dentista por não terem encontrado dentistas aderentes, o que, tendo em conta que a ARS LVT é a que tem menor rácio de dentistas aderentes, pode refletir uma baixa oferta de médicos aderentes. No entanto, é importante ter em conta que os principais motivos que levam os dentistas a não aderirem ao cheque-dentista em Portugal se devem principalmente ao valor reduzido do cheque-dentista, à demora no reembolso do valor dos cheques-dentista apresentados para pagamento e aos constrangimentos informáticos na apresentação dos cheques-dentista para pagamento. ${ }^{6}$

Os resultados obtidos mostram que é importante garantir o acesso efetivo dos alunos aos cheques-dentista, aumentar o número de dentistas aderentes, divulgar os locais onde se pode encontrar os dentistas aderentes e fornecer as informações sobre como utilizá-lo, sendo para tal importante a articulação com o Agrupamento de Escolas e os ACES. ${ }^{6,10}$

Apesar dos resultados obtidos, salienta-se a presença

Tabela 5 - Apresentação final da modelação multivariada

\begin{tabular}{lllll}
\hline Variável em análise & Categorias & Odds Ratio & IC (95\%) & Valor $\mathbf{p}$ \\
\hline Conhecimento de dentista aderente ao CD & $\begin{array}{l}\text { Não } \\
\text { Sim }\end{array}$ & 0,096 & $(0,047-0,198)$ & $<0,001$ \\
Vigilância por dentista particular & $\begin{array}{l}\text { Não } \\
\text { Sim }\end{array}$ & 2,004 & $(1,176-3,413)$ & 0,012 \\
\hline
\end{tabular}

CD: cheque-dentista 
dos seguintes vieses:

- Viés de seleção: a participação no estudo esteve numa primeira fase dependente da autorização prévia dos diretores das escolas/AE e depois da autorização do EE, sendo que os EE que consentiram em participar estarão à partida mais disponíveis ou interessados no problema em estudo. Apesar da escolha da amostra não ter sido aleatória, conseguiu-se uma boa participação no estudo e um número de participantes superior ao calculado teoricamente. Além disso, não se verificaram diferenças estatisticamente significativas entre o grupo de casos e controlos, no que diz respeito à caracterização sociodemográfica, o que nos pode fazer assumir a homogeneidade estatística dos grupos em comparação;

- Viés de informação: as respostas ao questionário são referentes a um tempo passado (ano letivo 2014/2015), o que pode ter levado a um viés de memória. Por outro lado, como o questionário não era validado, pode-se ter introduzido um viés de resposta. Para colmatar esta questão, foi realizado um pré-teste, para que fosse possível reajustar o questionário, no caso de alguma questão suscitar dúvidas.

Relativamente à validade externa, ou seja, a característica do estudo que permite generalizar os resultados do estudo a outras populações, é difícil tirar conclusões definitivas. Não obstante, a identificação dos motivos e fatores associados à não utilização do cheque-dentista, as conclusões podem ser generalizadas para a população-alvo, tendo em conta a metodologia usada e o tipo de estudo escolhido (amostra suficiente, relação caso-controlo), não sendo no entanto possível generalizar para outras populações diferentes.

\section{CONCLUSÃO}

Do nosso conhecimento, este é o primeiro estudo publicado que analisa os principais fatores associados à não utilização do cheque-dentista. O principal fator associado à não utilização foi o aluno ser vigiado por dentista particular e o principal fator associado à utilização foi o EE ter conhecimento de dentista aderente.

Uma das medidas previstas no Programa Simplex 2017 consiste na desmaterialização dos cheques-dentista, de forma a evitar o extravio e esquecimento do cheque e aumentar o uso dos cheques-dentista. ${ }^{27}$ Esta medida está prevista para o segundo trimestre de 2018, pelo que só vai ser operacionalizada nas escolas no ano letivo 2018/2019. Mesmo com a implementação desta medida, é importante aumentar a acessibilidade ao cheque-dentista. Apesar de se tratar de um estudo exploratório, com falta de suporte em estudos anteriores, os resultados obtidos têm plausibilidade e poderão ser úteis não só a nível local, mas também a nível nacional auxiliando no desenho de estudos e de intervenções em saúde que possam contribuir para melhorar a eficiência do PNPSO.

\section{PROTECÇÃO DE PESSOAS E ANIMAIS}

Os autores declaram que os procedimentos seguidos estavam de acordo com os regulamentos estabelecidos pelos responsáveis da Comissão de Ética da ARS LVT (número de autorização 9136/CES/ 2016), e de acordo com a Declaração de Helsínquia da Associação Médica Mundial.

\section{CONFIDENCIALIDADE DOS DADOS}

Os autores declaram ter seguido os protocolos do seu centro de trabalho acerca da publicação de dados.

\section{CONFLITOS DE INTERESSE}

Os autores declaram não haver conflitos de interesse.

\section{FONTES DE FINANCIAMENTO}

Este estudo não recebeu nenhuma bolsa ou financiamento do sector público, comercial ou sem fins lucrativos.

\section{REFERÊNCIAS}

1. Marcenes W, Kassebaum NJ, Bernabé E, Flaxman A, Naghavi M, Lopez A, et al. Global burden of oral conditions in 1990-2010: a systematic analysis. J Dent Res. 2013;92:592-7.

2. Petersen PE, Bourgeois D, Ogawa H, Estupinan-Day S, Ndiaye C. The global burden of oral diseases and risks to oral health. Policy Pract. 2005;83:661-9.

3. Patel R. The state of oral health in Europe: report commissioned by the Platform for Better Oral Health in Europe. Brussels: Platform for Better Oral Health in Europe; 2012.

4. Ministério da Saúde. Direção-Geral da Saúde. Programa Nacional de Promoção da Saúde Oral: Circular Normativa n: 01/DSE de 18 de janeiro. Lisboa: Direção-Geral da Saúde; 2005.

5. Calado R, Ferreira C, Nogueira P. Programa Nacional de Promoção da Saúde Oral: III Estudo Nacional de Prevalência das Doenças Orais 6, 12, 18, 35-44 e 65-74 anos. Lisboa: Direção-Geral da Saúde; 2015.

6. Entidade Reguladora da Saúde. Acesso, concorrência e qualidade no Programa Nacional de Promoção de Saúde Oral. Lisboa: Entidade Reguladora da Saúde; 2014.

7. Organisation for Economic Co-operation and Development. Health at a glance 2011: OECD indicators. Paris: OECD Publishing; 2011.

8. Ministério da Saúde. Direcção-Geral da Saúde. Alargamento do Programa Nacional de Promoção da Saúde Oral: grávidas e idosos beneficiários do complemento solidário: Circular Informativa no: O4/

DSPPS/DCVAE de 27 de fevereiro. Lisboa: Direção-Geral da Saúde; 2008.

9. Despacho n. ${ }^{\circ}$ 7402/2013. Diário da República n. ${ }^{\circ} 110$, Série II de 2013-06-07. Atribuição de cheques-dentista. Estabelece disposições referentes à atribuição de cheques-dentista. Lisboa: Assembleia da República; 2008. p. 1-2.

10. Circular Informativa: Saúde Oral - Normalização de procedimentos para a emissão de cheques-dentista e documentos de referenciação para higienista oral. Lisboa: Direção-Geral da Saúde; 2010. p. 1-16.

11. Lourenço A, Barros P. Cuidados de saúde oral - universalização. Lisboa: Nova Healthcare Initiative Research; 2016.

12. Júnior J, Gaspar AI, Vilela H, Filipe R. Plano Local de Saúde - ACES Lisboa Ocidental e Oeiras 2014-2016. Lisboa: ACES Lisboa Ocidental e Oeiras; 2015.

13. Perfil Local de Saúde do ACES Lisboa Ocidental e Oeiras 2016. Lisboa: Departamento de Saúde Pública da ARS LVT; 2017.

14. Unidade de Saúde Pública do ACES Lisboa Ocidental e Oeiras. Relatório de Atividades 2016. Lisboa: ACES Lisboa Ocidental e Oeiras; 2017.

15. de Silva AM, Hegde S, Akudo Nwagbara B, Calache H, Gussy MG, Nasser $M$, et al. Community-based, population level interventions for promoting child oral health. Cochrane Database Syst Rev. 2016;9:CD009837. 
16. Assembleia da República. Lei $n^{\circ} 67 / 98$, de 26 de outubro, com última alteração Lei n 103/2015, de 24 de agosto. Diário da república. Série I de 2011-03-02. Lei da Protecção Dados Pessoais (transpõe para a ordem jurídica portuguesa a Dir. n. ${ }^{\circ}$ 95/46/CE, do PE e do Conselho, 24/10/9. Lisboa: AR; 2015.

17. Dean AG, Sullivan KM. Tamanho da amostra: estudo de casos-controle não pareados. In: OpenEpi: Open Source Epidemiologic Statistics for Public Health: versão 301. OpenEpi; 2013.

18. Basílio N, Vitorino AS, Mendes Nunes J. Caracterização da empatia em internos de medicina geral e familiar. Rev Port Med Geral Fam. 2017;33:171-5.

19. Instituto Nacional de Estatística.Classificação Portuguesa 2010 das Profissões. Lisboa: INE; 2011.

20. Aguiar P. Estatística em investigação epidemiológica: SPSS: guia prático de dedicina. Lisboa: Climepsi Editores; 2007.

21. Ordem dos Médicos Dentistas. II Barómetro Nacional de de Saúde Oral de 2015. Lisboa: OMD; 2015.

22. Wall T, Nasseh K, Vujicic M. Most important barriers to dental care are financial, not supply related. American Dental Association Health Policy Institute (Research Brief). Chicago: ADA; 2014.

23. Organisation for Economic Co-operation and Development. Health at a glance 2015: OECD Indicators. Paris: OECD Publishing; 2015. p. 210.

24. Miller E, Lee JY, DeWalt DA, Vann Jr WF. Impack of caregiver literacy on children's oral health outcomes. Pediatrics. 2010;126:107-14.

25. Pedro AR, Amaral $O$, Escoval $A$. Literacia em saúde, dos dados à ação: tradução, validação e aplicação do European Health Literacy Survey em Portugal. Rev Port Saude Publica. 2016;34:259-75.

26. Cortés F, Rámon J, Cuenca E. Twelve years of the Infant Oral Care Program (PADI) in Navarra (1991-2002). Utilisation and health indicators. 2003;26:373-82.

27. Ministério da Presidência e da Modernização Administrativa. Programa SIMPLEX 2017. Lisboa: MPMA; 2017. 